\title{
Desarrollo de un sistema domótico con controlador difuso y controlador manual, implementado en LabView y Arduino IDE
}

\author{
José Alberto Vázquez Fernández, David Tinoco Varela \\ Universidad Nacional Autónoma de México, \\ Facultad de Estudios Superiores Cuautitlán, Departamento de Ingeniería, \\ Ingeniería en Telecomunicaciones, Sistemas y Electrónica, \\ México \\ itse.avazquez@gmail.com,dativa19@hotmail.com
}

\begin{abstract}
Resumen. En este trabajo se abordan los aspectos de diseño, implementación y experimentación de un sistema domótico, este esquema ha sido puesto en funcionamiento por medio de las herramientas de desarrollo LabView y Arduino IDE. El sistema busca gestionar aspectos comunes dentro de un hogar mediante controladores difusos y controladores manuales, con la intensión de que el inmueble diseñado pueda tener una respuesta automática, pero que también pueda ser controlado a gusto del usuario ante situaciones que salgan de lo cotidiano. En este sistema se considera la seguridad del inmueble mediante la implementación de una interfaz que pueda monitorizar la edificación a través de una cámara I P. Este trabajo fue realizado, buscando generar ambientes confortables, y agradables para un usuario. La estructura de la propuesta domótica se ha realizado en forma modular, para facilitar el análisis, la revisión y la reestructuración del sistema.
\end{abstract}

Palabras clave: Sistema domótico, HVAC, control difuso, interfaces.

\section{Development of a Home Automation System Using Fuzzy Controllers, and Manual Controllers, Implemented in LabView and Arduino IDE}

\begin{abstract}
In this work, the design, development, and implementation aspects of a home automation system are addressed, this scheme has been developed by means of the LabView and Arduino IDE development tools. The system seeks to manage common aspects within a home through fuzzy, and manual controllers, in order to the designed building can have an automatic response, but also it can be controlled at the user's convenience at situations different from everyday ones. The security of the building is considered through the implementation of an
\end{abstract}


interface which can monitor the building through an IP camera. This work was carried out, seeking to generate comfortable, and pleasant environments for the user. The structure of the house automation proposal has been made in a modular way, to facilitate the analysis, revision and restructuring of the system.

Keywords: Home automation system, HVAC, fuzzy control.

\section{Introducción}

El ser humano siempre ha buscado la forma de modificar su entorno, estas modificaciones son realizadas con la intención de generar un mayor grado de comodidad al existente. Las modificaciones incluyen, la generación de casas, o espacios donde descansar y realizar sus actividades de ocio y personales.

El avance de la tecnología y el uso de internet, puede permitir que una vivienda $u$ edificio pueda ser modificado de tal manera que un usuario pueda olvidarse de ciertas tareas rutinarias y permitir que la casa en sí misma, pueda realizar dichas tareas, tomando decisiones "propias". Este tipo de hábitats pueden ser considerados como casas inteligentes o sistemas domóticos.

Según la Asociación Española de Domótica e Inmótica, "un sistema domótico debe ser capaz de recoger información proveniente de sensores, procesarla y emitir órdenes" [1].

La domótica genera una integración dentro de un edificio u hogar de diferentes áreas del conocimiento, tales como las telecomunicaciones, la informática, la electrónica, y la inteligencia artificial. Estas estructuras son diseñadas con la finalidad de mejorar la calidad de vida de los usuarios, pero también se realizan buscando mejorar el ahorro energético y la protección al medio ambiente.

Un sistema domótico (SD) se compone de dos elementos, software y hardware. El hardware lo componen todos los dispositivos que se encargan de recibir señales del entorno, y efectuar cambios al entorno, como son los sensores y actuadores. El software por otro lado, es el responsable de analizar y procesar las señales de entrada, y gestionar las señales de salida.

Principalmente, se pueden distinguir dos tipos de arquitecturas de estos entes tecnológicos: la arquitectura centralizada, y la arquitectura distribuida.

En un sistema con arquitectura distribuida, cada dispositivo tiene un pequeño procesador propio que gestiona la información que se le ha sido pre programada por el fabricante, generando aplicaciones para funciones específicas. Este tipo de arquitectura, tiene la ventaja de que cada dispositivo cuenta con un alto nivel de autonomía, pero es debido a esta característica, que no se puede obtener una gran potencia del sistema, ya que todo el potencial está dividido en partes pequeñas.

Por otro lado, en la arquitectura centralizada, se tiene solamente un controlador, que es el encargado de recibir, procesar, y gestionar todas las señales de entrada y salida de un sistema. Al contrario de un sistema distribuido, su principal ventaja es el potencial de inteligencia que el sistema puede tener, sin embargo, si el procesador principal falla, todo el sistema falla. 
Estas estructuras se han presentado como el futuro de las viviendas y edificios, buscando que dichos inmuebles puedan conectarse directamente a internet, gestionando aspectos tales como las compras de consumibles y alimentos; buscando que se pueda regular el uso del consumo energético y el cuidado al medio ambiente; y obviamente, buscando que los usuarios se olviden de tareas rutinarias, y se genere un estado de confort y tranquilidad.

Es debido a las circunstancias mencionadas, que es de gran importancia tecnológica y comercial el desarrollo de sistemas domóticos que puedan ser implementados y modificados de una manera sencilla y económica.

En este proyecto se presenta un SD que ha sido diseñado con la finalidad de que pueda ser manejado, analizado y reestructurado de una manera sencilla. Esta característica estará determinada por el hecho de que el sistema se ha planteado de forma modular, donde cada módulo representara una de las funciones del diseño domótico, pudiéndose reestructurar (en caso de falla o mal funcionamiento) de manera individual, sin tener que alterar ningún otro modulo del sistema. La estructura planteada, gestiona aspectos tales como la temperatura, luminosidad y monitoreo de los interiores de un edificio, y por medio del control de estos aspectos, se busca generar un entorno agradable, fresco y seguro, sin importar los cambios climatológicos que puedan ocurrir en el exterior.

\section{Preliminares}

\section{a. Lógica difusa}

La lógica difusa surge entre los años 60's y 70's propuesta por Lofti A Zadeh [2]. Esta lógica permite simular los mecanismos de razonamiento humano para el control de sistemas, basados en la experiencia, proporciona un modelo matemático con el que se puede tratar la incertidumbre de los procesos cognitivos humanos y de este modo poder resolver problemas usando métodos matemáticos y computacionales.

Básicamente la lógica difusa es una forma de representar matemáticamente la incertidumbre y la vaguedad de un problema. Al buscar la solución del problema que fuere, se cuenta con variables de entrada y variables de salida, la lógica difusa permite la transformación entre las variables de entrada a su correspondiente salida privilegiando las características de significado en vez de las de precisión.

La lógica difusa emplea valores de números reales comprendidos entre 0 y 1 para indicar si un hecho es totalmente falso (0) o totalmente cierto (1), esto es el grado de pertenencia de un valor de entrada en un conjunto difuso, al ser multivaluada, una entrada puede pertenecer a más de un conjunto difuso al mismo tiempo, con un distinto valor de pertenencia para cada uno. Los conjuntos difusos, de este modo proporcionan una transición suave entre una característica y otra. Una vez que se han determinado los distintos valores de pertenencia de una entrada a cada uno de los conjuntos en el universo de discurso se dice que la variable se ha borrosificado. 


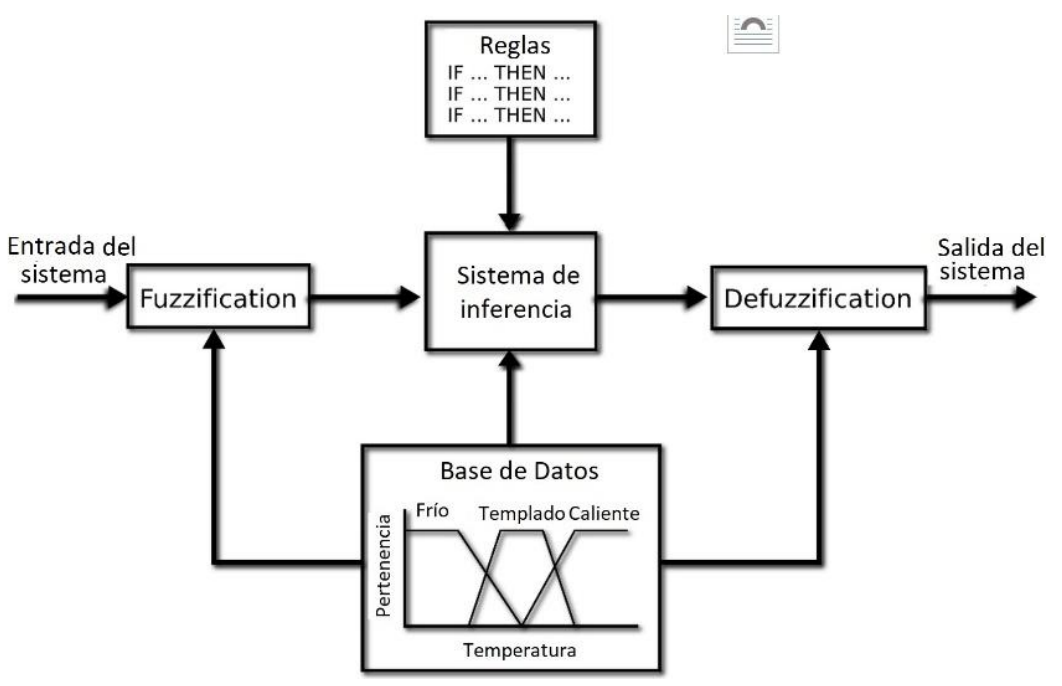

Fig. 1. Esquema básico de un sistema de control difuso.

A partir de la lógica difusa, se han generado los denominados controladores difusos, sistemas que han sido eje principal de desarrollo de una gran cantidad de aplicaciones e interfaces $[3,4]$. Un sistema de control estándar basado en lógica difusa se puede representar de acuerdo a la figura 1.

\section{b. Arduino}

Según la página oficial del proyecto Arduino (https://www.arduino.cc/), Arduino es una plataforma electrónica de código abierto basada en hardware y software de fácil manejo. Las placas Arduino pueden leer entradas por medio de sensores y botoneras, procesar estas entradas, y generar controles de diferentes tipos de actuadores en sus salidas.

Esta tarjeta de operaciones, se ha convertido en el proceso central de miles de proyectos, que van de proyectos muy simples a proyectos científicos sumamente complejos.

Arduino fue desarrollado en el Ivrea Interaction Design Institute como una herramienta que pudiera ser usada fácilmente por cualquier persona sin experiencia en electrónica y programación para la generación de prototipos tecnológicos. Existen varias versiones de la placa Arduino, sin embargo, la placa utilizada en este proyecto ha sido la placa UNO. 


\section{Estado del arte}

Muchos trabajos de implementaciones y propuestas domóticas se han presentado en la literatura científica, tratando principalmente temas relacionados al confort dentro de un edificio y al cuidado ambiental, dejando a un lado la facilidad de desarrollo, trabajos que en muchos casos utilizan la lógica difusa para su implementación. En esta sección solamente mencionaremos algunos de ellos y sus principales características, para dar un panorama general del desarrollo de este tipo de sistemas.

Existen implementaciones domóticas muy interesantes desde un punto de vista científico, por ejemplo, Pardo, Strack y Martínez en [5] presentaron un sistema para controlar dispositivos domésticos utilizando servicios web y una conexión doméstica común a Internet. Ellos mencionan que los dispositivos se pueden conectar a una computadora en el hogar y se pueden manipular o programar local o remotamente accediendo a un servidor web, lo que permite la independencia entre la aplicación local que controla los dispositivos desde el hogar y el servidor que permite el acceso remoto.

En [6], Rodríguez, Piedra e Iribarne, realizaron un sistema de aprendizaje que recopila información de la interacción del usuario con el sistema, y genera las reglas correspondientes que definirán el conocimiento del entorno domótico. Los autores mencionan que en su trabajo, ellos agregan la novedad de que las reglas que definirán el proceso de adaptación no son estáticas y preestablecidas, sino que pueden modificarse a lo largo del tiempo.

Una propuesta por demás interesante, es la presentada por Ché y Pardons, en [7], donde se menciona que el objetivo en una casa inteligente es aprender, reconocer y automatizar los patrones de interacción entre un individuo y los diversos dispositivos de automatización del hogar. Los autores consideran este ejemplo: el patrón es despertarse, apagar el despertador y luego hacer café. Un sistema de aprendizaje inteligente podría aprender este patrón y automáticamente preparar café por la mañana, cuando la persona apaga el despertador.

Como ya se mencionó, la generación de sistemas más amigables con el ambiente es uno de los principales objetivos de los sistemas domóticos, tal como lo muestran Villar, De La Cal y Sedano en [8]. Ellos presentan una solución de sistema multi agente para la reducción del consumo de energía en sistemas de calefacción de casas. En su propuesta se tiene una unidad central de control (CCU) responsable de minimizar el consumo de energía que interactúa con los calentadores. La CCU incluye un modelo difuso y un controlador difuso, y hace uso del concepto de balance de energía para distribuir la energía entre los calentadores.

Los proyectos domóticos, no solo buscan cuidar aspectos ambientales y de confort, también buscan generar espacios que puedan permitir el mejoramiento de la calidad de vida [9].

Por otro lado, Arduino ha jugado un papel importante en el caso de este tipo de sistemas, comportándose como parte principal en muchos de ellos. Por ejemplo, en [10] se diseñó e implemento una casa inteligente remotamente controlable, energéticamente eficiente y altamente escalable con características básicas que protegen la comodidad y la seguridad de los residentes. En este caso, los autores realizaron una red doméstica, por medio de sensores y actuadores. En su proyecto, la placa Arduino funciono como 
procesador central de la red. Arduino también sirvió como punto de comunicación entre el sistema diseñado y una aplicación Android. De manera similar, en [11] fue mostrado un sistema de control de hogar y control ambiental de bajo costo y flexible. Los autores emplearon un micro servidor web integrado en el microcontrolador Arduino Mega 2560, con conectividad IP para acceder y controlar dispositivos de forma remota.

Chandramohan y otros autores en [12] presentaron un sistema de monitoreo y control hogareño económico y flexible con la ayuda de un servidor micro-web integrado con conectividad de protocolo de internet (IP) para acceder y controlar equipos y dispositivos remotamente, usando una aplicación de teléfono inteligente basada en Android. En este proyecto, la placa Arduino funciono como operador principal de todo el sistema, recibiendo la información de los sensores y generando la comunicación con el dispositivo Android definido.

Como se ha podido leer, la placa Arduino ha sido pieza importante en diversos sistemas domóticos, sin embargo, en los sistemas mencionados la interfaz esta definida por medio de una aplicación en el sistema operativo Android, caso contrario a la propuesta presentada en este trabajo, dónde la interfaz de usuario estará gestionada por LabView.

\section{Desarrollo del proyecto}

En esta sección se describe el proceso de desarrollo del proyecto, el sistema difuso utilizado, y la generación de la interfaz desarrollada por medio de LabView y sus instrumentos virtuales (VI) para generar el control de nuestro SD.

El SD está desarrollado en función de un sistema tipo HVAC (Heating, Ventilating and Air Conditioning), un controlador difuso, controladores on/off, controladores de luminosidad, así como un sistema de cámara IP. La plataforma en la cual se desarrolló el sistema completo es LabVIEW de National Instruments NI, obteniendo información de la placa Arduino. En esta sección, se describirá cada uno de los elementos que conforman al SD presentado.

\section{a. Esquema general}

El sistema se ha planteado como un proceso modular, en donde cada módulo es independiente de los demás en su análisis, implementación, diagnóstico y reestructuración, sin embargo, todos estos módulos son monitorizados y pueden ser controlados manualmente por medio de una interfaz realizada en LabView. El sistema propuesto consta de una serie de tarjetas de desarrollo Arduino que leerán la información de los sensores y enviarán las señales de control a los elementos de salida. La comunicación entre las tarjetas y la computadora que ejecuta LabView, se realiza por medio del puerto serial. En el esquema se tienen los valores de entrada: sensor de temperatura, sensor de humedad, sensor de luz, sensor de presencia, código de acceso vía Wi-Fi para el inicio de la cámara IP y los botones virtuales para controlar los focos en forma On-Off. Como elementos de salida se tiene un ventilador (controlado por medio de un sistema difuso), un calefactor (controlado por medio de un sistema difuso), 


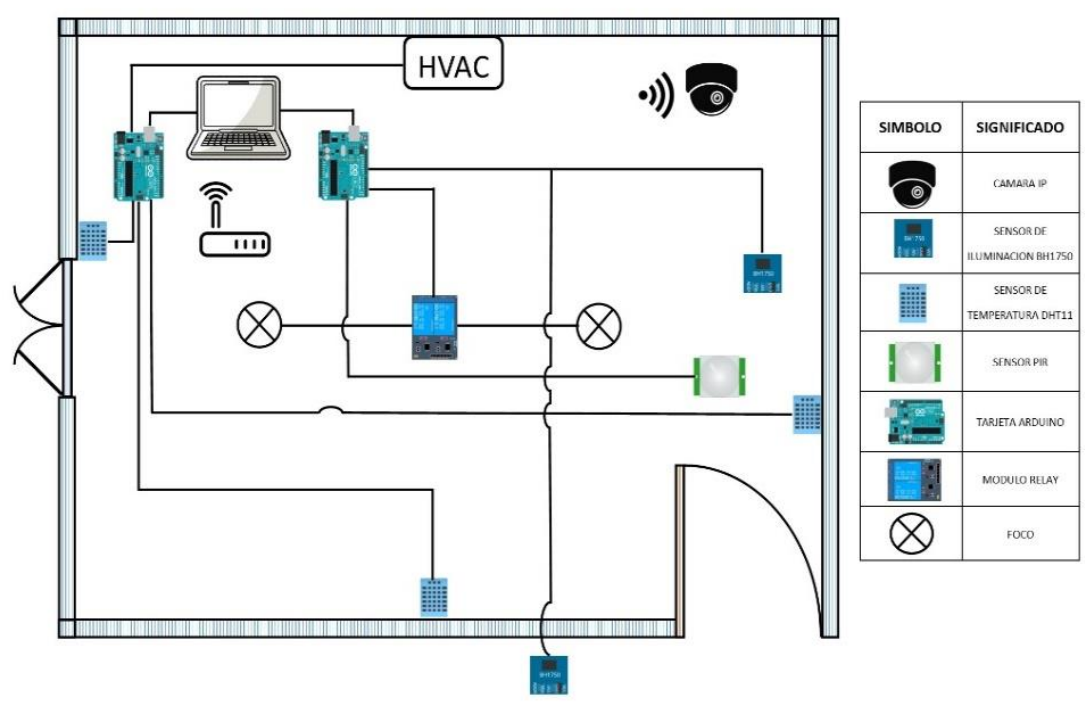

Fig. 2. Esquema general de conexiones de entradas y salidas del sistema propuesto, donde HVAC representa al ventilador y al calentador.

las señales que las luces recibirán, y la activación de la cámara IP. Todos estos dispositivos fueron implementados en una maqueta, la estructura del diseño propuesto de la maqueta se puede ver en la figura 2 . El esquema de software que engloba todo el procesamiento de los datos, puede observarse en la figura 3.

\section{b. Selección de herramientas a utilizar}

Existen en el mercado una gran cantidad de tarjetas de desarrollo que puede ser utilizadas para el diseño de prototipos y proyectos tecnológicos, entre ellas están RaspBerry Pi (https://www.raspberrypi.org/), Beagle Bone (http://beagleboard.org/bone) y Arduino. Cada una con sus características particulares, estas características pueden observarse en la tabla 1.

En la tabla 1, podemos observar que tarjetas como Raspberry Pi y BeagleBone, tienen mayores tamaños de memoria, y son mucho más rápidas que un Arduino UNO, sin embargo, estas tarjetas están principalmente enfocadas a software, a diferencia de la tarjeta Arduino que se enfoca a hardware. Por este motivo, esta tarjeta está más cercana a las necesidades que se tienen para el proyecto planteado, ya que lo que se busca es la interacción de elementos de hardware con la interfaz de LabView. La tarjeta Arduino es la tarjeta más económica en el mercado, lo que la hace accesible a casi cualquier sector poblacional. A pesar de las ventajas ya mencionadas, otra circunstancia por la que se ha elegido a Arduino sobre las otras tarjetas, es por la compatibilidad que esta tarjeta tiene con prácticamente cualquier sistema, incluyendo una compatibilidad directa con LabView. 


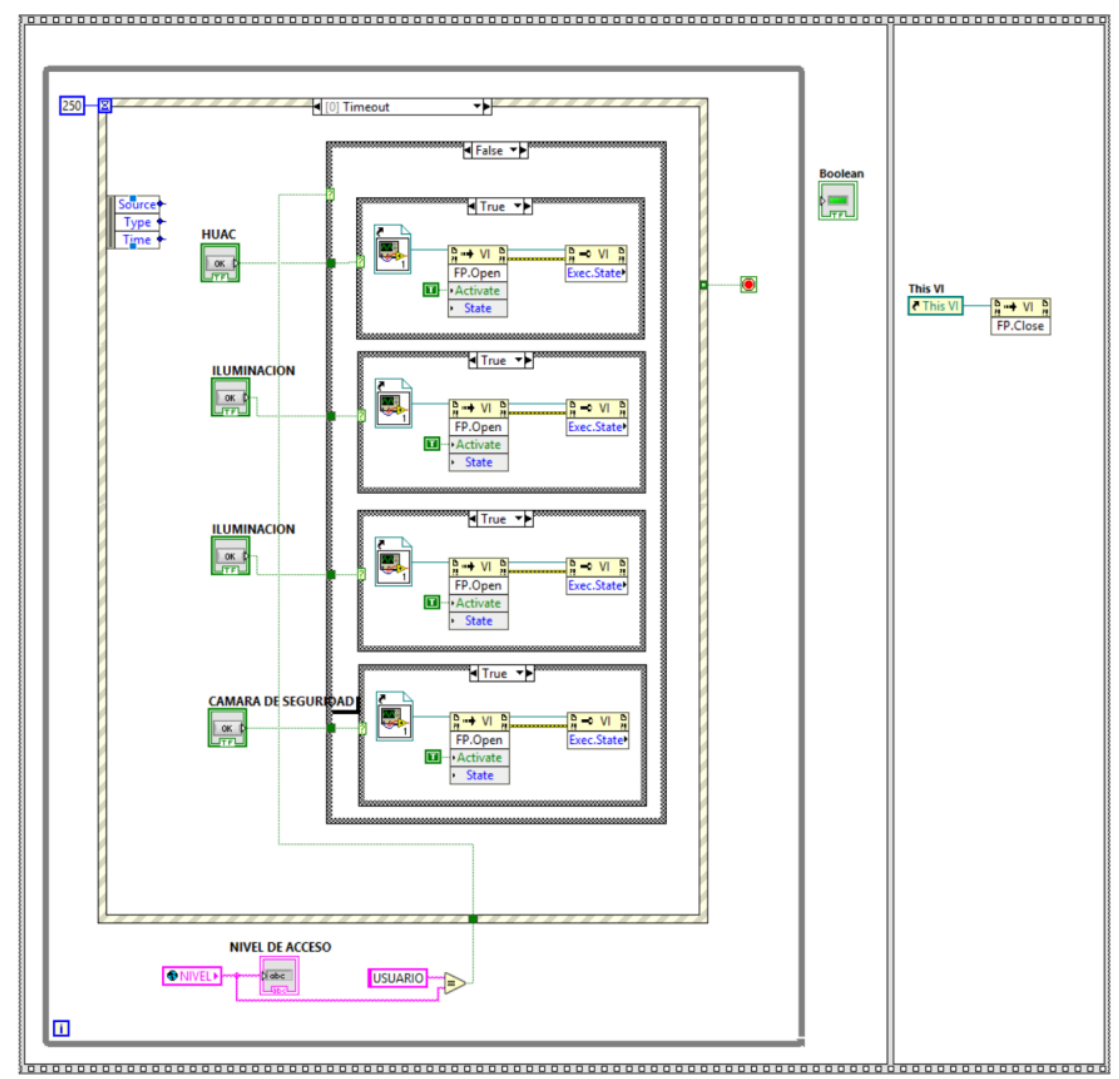

Fig. 3. Esquema general del software que procesa y gestiona las señales de entrada y salida del SD propuesto.

Esta situación permite que se pueda utilizar este tipo de tarjeta bajo distintos esquemas, situación que no presentan de manera directa las demás tarjetas de desarrollo. Principalmente se ha usado Arduino por su facilidad de reconfiguración.

\section{c. Control de iluminación}

Este control está enfocado a generar estancias que tengan un buen nivel de iluminación, iluminación que permitirá a los usuarios del edificio, o casa, sentirse cómodos bajo estas circunstancias lumínicas. Al generar un control inteligente de encendido de luces, se logra que este ambiente agradable, se pueda mantener sin importar el horario o las circunstancias existentes en el entorno.

La gestión del control de iluminación dentro del sistema planteado se realiza mediante un controlador que toma como variables principales los datos obtenidos de sensores BH1750 (sensor de luz) dentro y fuera de la habitación, el uso principal que tiene la habitación, y la presencia de alguien en la habitación. 


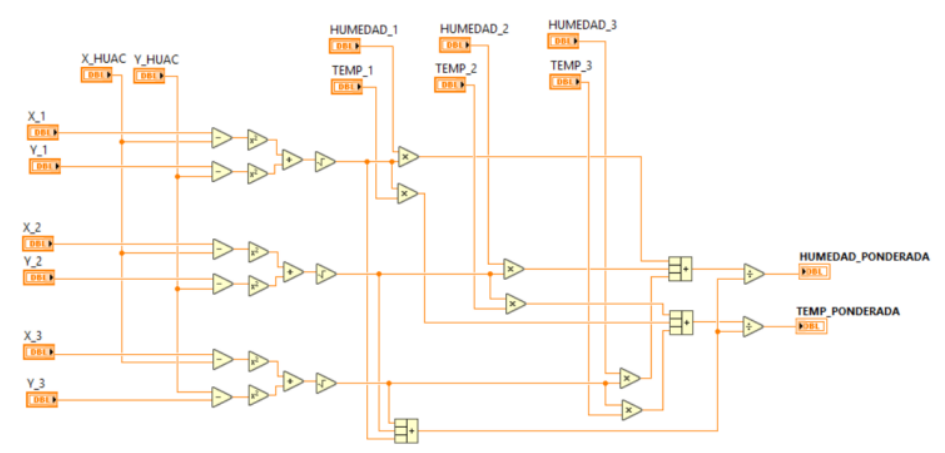

Fig. 5. Ponderación de las mediciones obtenidas por medio de los sensores.

Tabla 2. Reglas de evaluación para las entradas de temperatura y humedad, y su evaluación para la salida controlada por niveles para el ventilador.

\begin{tabular}{cccc}
\hline HUMEDAD & BAJA & MEDIA & ALTA \\
\hline TEMPERATURA & & & N0 \\
\hline MUY FRIO & $\mathrm{N} 0$ & $\mathrm{~N} 0$ & $\mathrm{~N} 1$ \\
\hline FRIO & $\mathrm{N} 0$ & $\mathrm{~N} 0$ & $\mathrm{~N} 2$ \\
\hline TEMPLADO & $\mathrm{N} 0$ & $\mathrm{~N} 0$ & $\mathrm{~N} 4$ \\
\hline CALIENTE & $\mathrm{N} 2$ & $\mathrm{~N} 3$ & $\mathrm{~N} 5$ \\
\hline MUY CALIENTE & $\mathrm{N} 3$ & $\mathrm{~N} 4$ &
\end{tabular}

El controlador desarrollado en LabView toma estos 3 valores por medio de una tarjeta Arduino, los evalúa y define la cantidad lumínica apropiada para la habitación, es obvio mencionar que si, por ejemplo, no hay luz en la habitación, pero tampoco existe una presencia en ella, el controlador definirá que no es necesario encender los focos de dicha habitación. El diagrama de bloques que muestra este controlador se observa en la Fig. 4.

\section{d. Control de temperatura}

El sistema de control de temperatura está enfocado principalmente a brindar confort de los usuarios, manteniendo una temperatura adecuada en cada momento para las personas que se encuentren dentro del inmueble domótico, controlando también, las temperaturas en zonas particulares del edificio.

El Instituto para la Diversificación y Ahorro de la Energía (IDAE) indica que la temperatura ideal para sentir confort va de los 19 a los 21 grados en una habitación o casa, sin embargo, también mencionan que una temperatura de $21^{\circ} \mathrm{C}$ es suficiente para 
Tabla 1. Principales características de distintas tarjetas de desarrollo.

\begin{tabular}{lccc}
\hline \multicolumn{1}{c}{ Características } & Raspberry Pi 3 & BeagleBone & Arduino UNO \\
\hline Memoria & $1.0 \mathrm{~GB}$ & $512 \mathrm{MB}$ & $2 \mathrm{~KB}$ \\
\hline $\begin{array}{l}\text { Velocidad de } \\
\text { procesamiento }\end{array}$ & $1.2 \mathrm{Ghz}$ & $1.0 \mathrm{GHz}$ & $16 \mathrm{MHz}$ \\
\hline Pines de entrada-salida & 40 & 92 & 14 \\
\hline Puertos USB & 2 & 1 & 1 \\
\hline Precio & $53 \mathrm{USD}$ & $85 \mathrm{USD}$ & $20 \mathrm{USD}$ \\
\hline
\end{tabular}

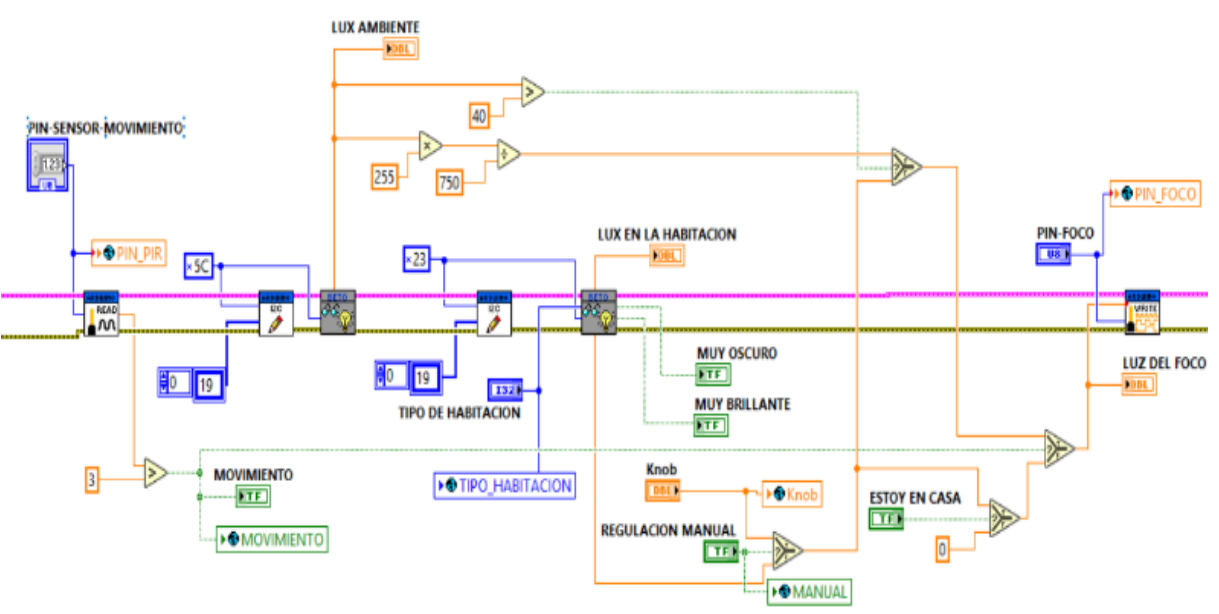

Fig. 4. Diagrama principal para la gestión de iluminación.

mantener el confort de una vivienda [2], aunque dependerá también de la cantidad de gente que se encuentre en ella.

Para el control de temperatura se utilizó la funcionalidad de un sistema HVAC. Una placa Arduino se encarga de recolectar los datos de los sensores de temperatura y humedad. Estos datos son enviados a un controlador difuso diseñado en LabView. El sistema difuso regresa los valores de respuesta hacía dos salidas: el encendido en diferentes potencias de un ventilador, y el apagado y encendido de un calefactor.

El sistema para el control de la temperatura consta de varias partes entre las principales se encuentran: Lectura de sensores, ponderación de mediciones y controladores difusos.

La ponderación es algo importante en un sistema HVAC ya que se toma en cuenta la posición del sensor con respecto al sistema de ventilación para así darle un valor de importancia más certero al momento de establecer la temperatura y humedad del lugar. El cálculo de la ponderación necesaria para cada uno de los sensores puede verse en la figura 5.

En este módulo se utilizó un etiquetado (generación de conjuntos difusos) para el manejo de los niveles de temperatura y humedad, el etiquetado y acciones ante ciertos niveles se manejaron mediante controladores difusos, los cuales nos facilitan la toma de decisiones y brindan una mayor certidumbre al usuario. El controlador fue diseñado con la herramienta Fuzzy System Designer que ofrece LabView. 
Desarrollo de un sistema domótico con controlador difuso y controlador manual, implementado ...
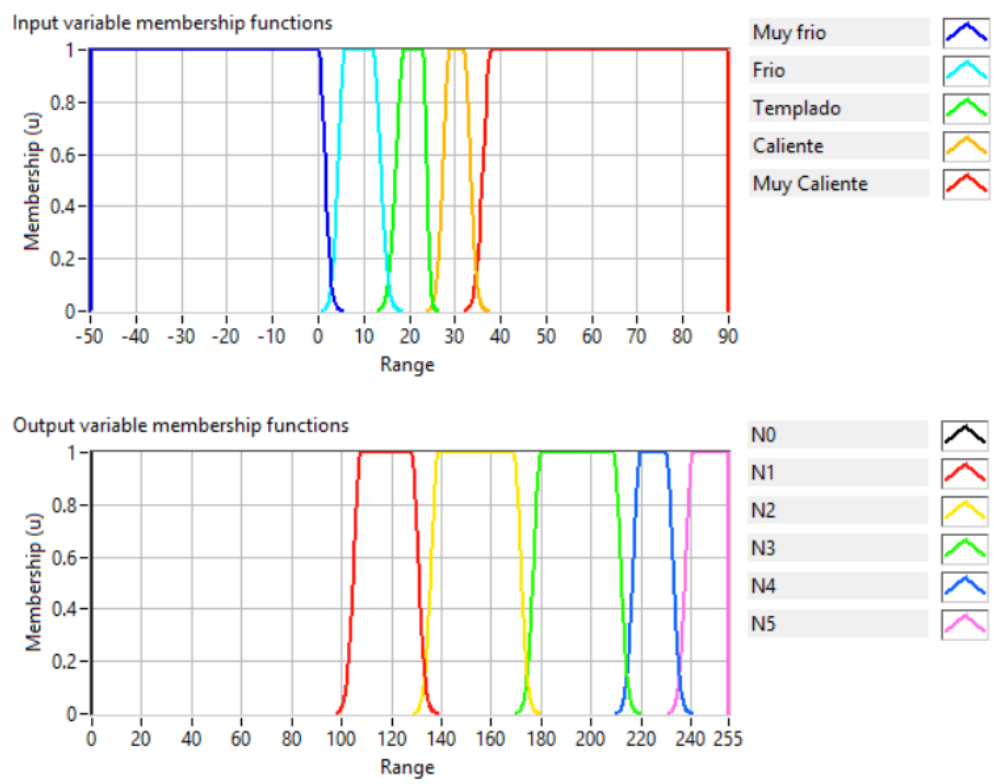

Fig. 6. Conjuntos difusos diseñadas para las variables de entrada (temperatura y humedad) y de salida (PWM y calefactor).

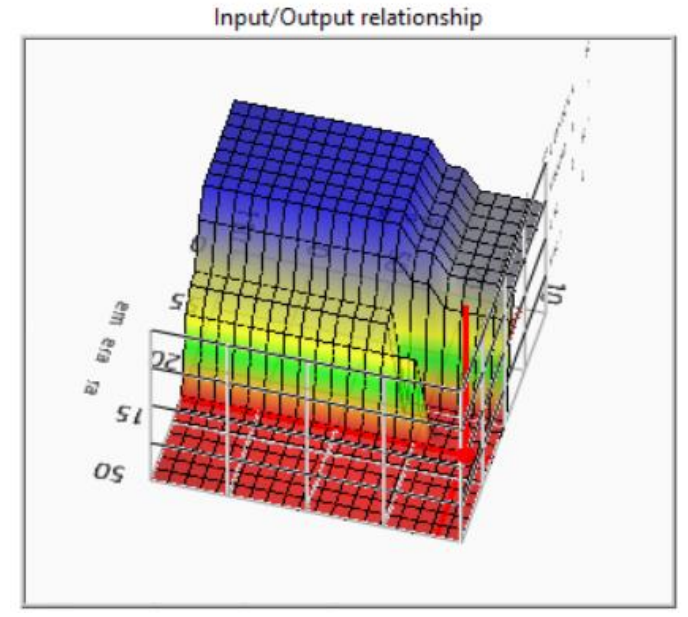

Fig. 7. Comportamiento general del sistema difuso, donde se pueden simular diferentes valores de entrada de humedad y temperatura.

Las variables de entrada a considerar para este controlador son la temperatura y la humedad, mientras que en las variables de salida se tiene el PWM (Pulse Width Modulation) que controla la potencia del ventilador; y también se considera el encendido y apagado de un calefactor como salida 2. Los conjuntos difusos generados para el control del ventilador y del calefactor pueden verse en la figura 6 . 


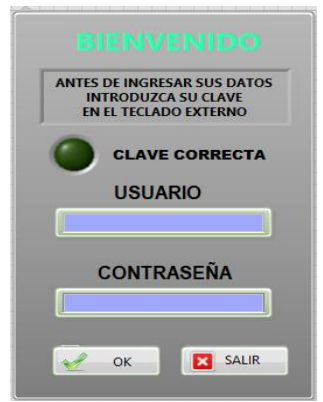

Fig. 8. Interfaz de login.

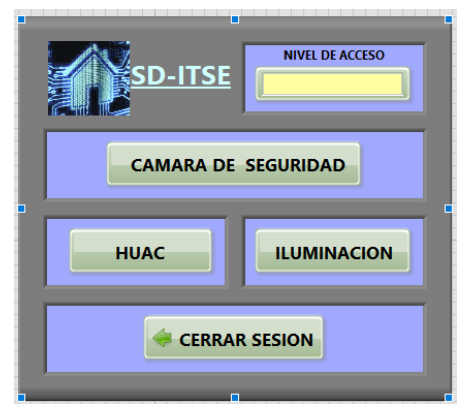

Fig. 9. Panel de control de aplicaciones del sistema.

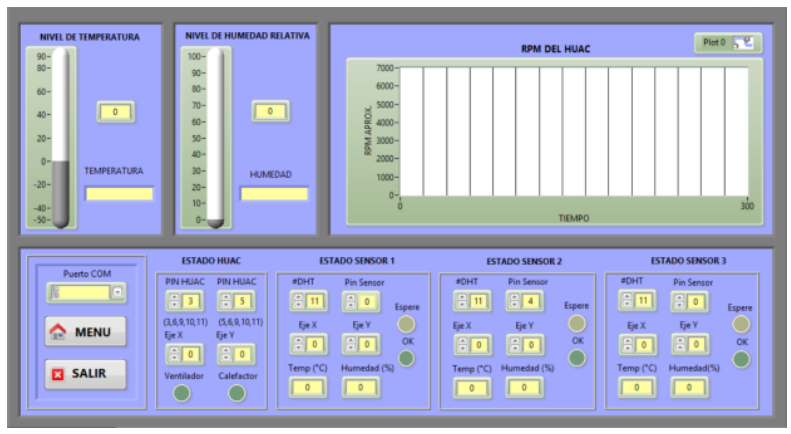

Fig. 10. Panel de administrador del módulo HVAC.

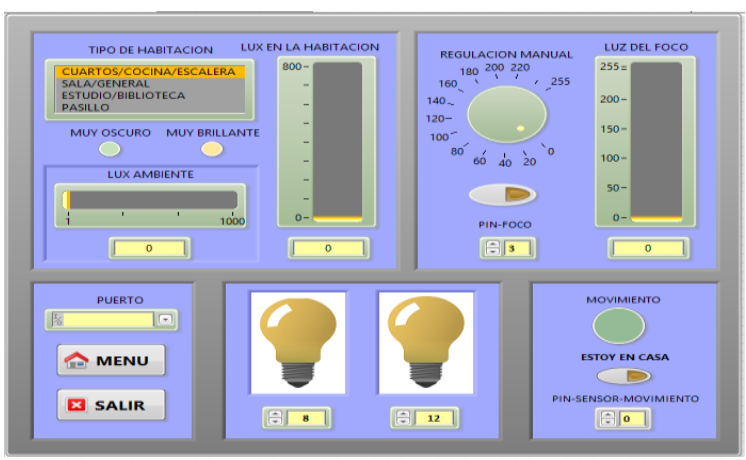

Fig. 11. Panel de usuario del módulo de luminosidad.

El establecimiento de las reglas para la evaluación de las variables de entrada, se realizaron tomando en cuenta los efectos que tienen los niveles de humedad en un ambiente, y con esto establecer la velocidad con la que funciona el ventilador y de igual forma si se enciende o mantiene apagado el calefactor. En la tabla 2 se observa el conjunto de reglas que rigen el controlador difuso.

La herramienta de diseño difuso de LabView, nos permite visualizar el comportamiento general del funcionamiento del controlador, y ver su respuesta ante 
Desarrollo de un sistema domótico con controlador difuso y controlador manual, implementado ...

valores de entrada dados por el sensor de humedad y de temperatura. Dicho comportamiento puede verse en la fig. 7 .

\section{e. Video vigilancia}

La idea general de un sistema de cámaras remoto en el caso de la domótica, es permitir al usuario monitorear a larga distancia los cambios o eventualidades que sucedan tanto al interior como al exterior de un hogar, tal que solo una persona autorizada pueda acceder y observar a través de dichas cámaras desde un punto remoto.

Este módulo dentro del SD, tiene como objetivo el poder generar un sistema de seguridad utilizando cámaras IP, con la intención de brindar no solo comodidad sino también seguridad de forma remota al esquema propuesto.

Para la implementación del sistema se utilizó la cámara de un Smartphone, lo que es un ejemplo de la ganancia económica que puede ofrecer el sistema, al no necesitar utilizar dispositivos especiales.

\section{f. Interfaz del sistema domótico}

La interfaz del SD, se realizó considerando un ambiente amable con el usuario y fácil de utilizar. Esta interfaz contiene una etapa de acceso, cuando se inicia la aplicación el sistema manda una interfaz de login en el cual se ingresan un nombre de usuario y contraseña para la identificación de usuarios y administradores, esto tiene como finalidad el establecer un nivel de acceso. El ingreso al sistema puede verse en la figura 8.

En caso de que tanto el usuario como la contraseña estén registrados en la base de datos del sistema, se mostrara la ventana de la figura 9 donde se pueden elegir las aplicaciones a las que se desea ingresar y también se mostrara el nivel de acceso.

En caso ingresar al módulo del HVAC (control de temperatura) en modo de administrador, se mostrará la interfaz de la Fig. 10. En este módulo podemos controlar la asignación de los puertos de los sensores y el ventilador. Al iniciar la aplicación, la interfaz nos arrojara las temperaturas que se obtengan de los sensores, la humedad relativa y las revoluciones del motor del ventilador, todo esto gestionado por medio del controlador difuso.

En caso de ingresar como usuario, solamente se permite observar la temperatura, humedad relativa, la activación del ventilador y que sensores se encuentran realizando mediciones.

Cuando se ingresa como administrador en el módulo de iluminación, se despliega la pantalla de la Fig. 11, en este módulo se conjuntan el controlador On/Off y el control de luminosidad. En la aplicación podemos controlar el encendido y apagado de los focos, así como la asignación de los puertos donde se controla cada uno, podemos controlar la iluminación de manera manual y observar la intensidad de luz que emiten los focos, se puede elegir el tipo de habitación en la cual están colocados los sensores, ver la iluminación de la habitación y observar si existe alguna presencia dentro de ella. En el acceso de usuario, solamente se permite usar el controlador On/Off de los focos, 
observar los datos obtenidos por los sensores de luminosidad y controlar la cantidad de luz que emiten los focos con la regulación manual.

Por último, si se ingresa a la aplicación de cámara de seguridad la aplicación abrirá una pantalla, en donde se muestra el video que se recibe a través de un dispositivo móvil, el sistema permite monitorear una cámara que esté conectada a la misma red que la computadora donde se esté corriendo la aplicación del SD.

\section{g. Evaluación del sistema propuesto}

Como ya se ha mencionado, se generó un sistema en LabView que obtiene la información proveniente de los Arduinos por comunicación serie, procesa la información, calcula las salidas, y envía estos datos de regreso a las placas Arduino. El sistema físico se implementó en una maqueta, donde fueron instalados todos los sensores y elementos de salida ya descritos.

La maqueta física fue sometida a diferentes cambios de temperatura y humedad, cambios generados por calentadores, encendedores, $\mathrm{y}$ atomizadores de agua. El programa realizado en LabView, muestra en tiempo real los valores de salida que el sistema difuso va calculando, con estos valores en pantalla es fácil observar, la velocidad con la que el ventilador trabaja, y los momentos en los que el calentador es activado o desactivado. Las pruebas realizadas en este módulo, lograron generar ambientes confortables, estabilizando una temperatura promedio de $21^{\circ} \mathrm{C}$ dentro de todas las secciones de la maqueta.

El control lumínico del sistema se lleva a cabo en dos formas, por un lado, LabView recibe la información proveniente de los sensores de luz y de presencia, y en base a los valores obtenidos, realiza el cálculo para definir el mejor nivel de iluminación. Las pruebas realizadas mostraron que de esta manera se evitaba realizar un gasto innecesario de luz, cuando no hay una presencia en la habitación de la maqueta o cuando había suficiente luz externa dentro de las habitaciones, el sistema no genera emisión lumínica, sin embargo, a pesar del gasto energético que se puede ahorrar, el sistema cuenta con una interfaz que permite a un usuario autorizado encender las luces sin importar las demás variables a evaluar. Esta es una característica importante del sistema, ya que permite al usuario poder controlar el inmueble cuando las circunstancias salen de lo cotidiano.

Una de las principales ventajas de esta propuesta, es la posibilidad de reestructurar cualquier módulo de forma independiente, sin necesidad de alterar o siquiera tener que ingresar en cualquier otro modulo. Esta circunstancia permite la libertad de acción sobre cualquier modulo, sin considerar que pueda alterarse cualquier otro sub sistema. Si un módulo se estropea, solo se reestructura ese modulo, y el resto del SD permanece funcionando de manera normal.

Una de las principales cualidades de la estructura planteada, es la posibilidad de poder elegir entre los tipos de control automático y manual por medio de la interfaz en LabView. Esto permite que el usuario pueda ajustar el comportamiento de su sistema a su entero agrado y conveniencia. Si existe una circunstancia que salga del comportamiento usual del usuario tal como una enfermedad, él podrá solamente tomar el control del edificio y definir las mejores condiciones en ese instante. 
El inmueble planteado, ha mostrado funcionar de manera adecuada a las pruebas realizadas, y como ventaja principal, este esquema puede diagnosticarse y modificarse de manera simple, debido a que se ha desarrollado como un conjunto de módulos.

\section{Conclusiones}

En la búsqueda de un nivel de automatización optimo en los hogares, oficinas, edificios y/o lugares públicos es necesario integrar diversas áreas de la ingeniería, física y de la salud para lograr desarrollar un sistema que brinde la comodidad adecuada en nuestro entorno, y a la vez la seguridad de que el sistema está trabajando "pensando" en las necesidades del usuario.

En este trabajo, se ha presentado una opción de ambiente domótico que es una opción económica en hardware dentro del ámbito de la automatización de viviendas. En este proyecto, se han utilizado componentes y herramientas conocidas como puntos de soporte, tal como la placa de desarrollo Arduino y la plataforma LabView.

Con la intención de facilitar el desarrollo y manejo interno del proyecto, se planteó una estructura e implementación en una forma modular, por lo cual cada módulo puede ser añadido, eliminado o modificado sin afectar a todo el sistema en general. Esta es una de las principales ventajas de la propuesta, ya que, si una sección del sistema falla, esa falla no se propagará a ninguna otra sección, permitiendo identificar y solucionar rápidamente el problema.

Este sistema fue planteado utilizando una combinación de diferentes formas de control, sin embargo, la utilización del controlador difuso, permite generar un ambiente completamente independiente y que responde a las necesidades para las que fue diseñado, es decir, permite dar comodidad a un usuario sin que él se dé cuenta de que la climatización del edificio o vivienda se está modificando constantemente, obviamente de manera precisa a sus necesidades.

Por otro lado, el esquema planteado permite poder modificar el comportamiento de los elementos de salida de manera manual, esta es otra gran ventaja que le permite al usuario tener un control total de su ambiente, aun cuando situaciones diferentes a las cotidianas sucedan.

Para este sistema también se generó una interfaz que es amable con el usuario y que permite facilidad de entendimiento, esta interfaz se dividió en dos tipos de acceso, el de administrador y el de usuario común, para que el acceso al sistema tenga jerarquía y se pueda mantener un control adecuado del funcionamiento del sistema.

Agradecimientos. Este trabajo fue en parte financiado por el proyecto PAPIIT IN 113316, y el proyecto PIAPI 1634 de la UNAM.

\section{Referencias}

1. Asociación Española de Domótica e Inmótica: ¿Qué es domótica? (2017)

2. Zadeh, L.A.: Fuzzy sets. Information and control, 8(3), pp. 338-353 (1965) 
3. Pires, G., Nunes, U.: A wheelchair steered through voice commands and assisted by a reactive fuzzy-logic controller. Journal of Intelligent \& Robotic Systems, 34(3), pp. 301314 (2002)

4. Ye, C., Yung, N.H., Wang, D.: A fuzzy controller with supervised learning assisted reinforcement learning algorithm for obstacle avoidance. IEEE Transactions on Systems, Man, and Cybernetics, Part B (Cybernetics), 33(1), pp. 17-27 (2003)

5. Pardo, M.E., Strack, G., Martínez, D.C.: A domotic system with remote access based on web services. Journal of Computer Science \& Technology, 8 (2008)

6. Rodríguez-Garcia, D., Piedra-Fernández, J. A., Iribarne, L.: Adaptive Domotic System in Green Buildings. In Advanced Applied Informatics (IIAI-AAI), IIAI 4th International Congress on, IEEE. pp. 593-598 (2015)

7. Ché, N.K., Pardons, N., Vanrompay, Y., Preuveneers, D., Berbers, Y.: An intelligent domotics system to automate user actions. In: Ambient Intelligence and Future TrendsInternational Symposium on Ambient Intelligence (ISAmI’10) pp. 201-204, Springer, Berlin (2010)

8. Villar, J.R., De La Cal, E., Sedano, J.: Energy saving by means of fuzzy systems. In: International Conference on Intelligent Data Engineering and Automated Learning, Springer, pp. 155-167 (2007)

9. Vacher, M., Istrate, D., Portet, F., Joubert, T., Chevalier, T., Smidtas, S., Méniard, S.: The sweet-home project: Audio technology in smart homes to improve well-being and reliance. In: Engineering in Medicine and Biology Society, (EMBC'11) Annual International Conference of the IEEE, pp. 5291-5294 (2011)

10. Baraka, K., Ghobril, M., Malek, S., Kanj, R., Kayssi, A.: Low cost arduino/android-based energy-efficient home automation system with smart task scheduling. In: Computational Intelligence, Communication Systems and Networks (CICSyN'13) Fifth International Conference on. IEEE, pp. 296-301 (2013)

11. David, N., Chima, A., Ugochukwu, A., Obinna, E.: Design of a home automation system using arduino. International Journal of Scientific \& Engineering Research, 6(6), pp.795801 (2015)

12. Chandramohan, J., Nagarajan, R., Satheeshkumar, K., Ajithkumar, N., Gopinath, P.A., Ranjithkumar, S.: Intelligent smart home automation and security system using Arduino and Wi-fi. International Journal of Engineering and Computer Science, 6(3) (2017) 